\title{
AUTOMATIC CONTINUITY AND OPENNESS OF CONVEX RELATIONS
}

\author{
J. M. BORWEIN
}

\begin{abstract}
We establish open mapping and lower semicontinuity results for convex relations which are generalizations of classical results on continuity of positive linear mappings. These results do not require closure of the relation. Several applications are given.
\end{abstract}

1. Preliminaries. Let $X$ and $Y$ be (separated, real) topological vector spaces. A relation $H: X \rightarrow Y$ is convex if its graph $\operatorname{Gr}(H):=\{(x, y) \mid y \in H(x)\}$ is convex in $X \times Y$. The relation is a convex process if $\operatorname{Gr}(H)$ is actually a convex cone. We denote the domain by $D(H):=\{x \mid H(x) \neq \varnothing\}$ and the range by $R(H):=$ $\{y \mid y \in H(x), \exists x \in X\}$. The inverse relation is defined by $x \in H^{-1}(y)$ if $y \in H(x)$, and has $R\left(H^{-1}\right)=D(H)$. For any set $C$ in $X, H(C):=\{H(x) \mid x \in C\}$ so that $R(H)=H(X)$.

A relation $H$ is $L S C$ at $x$ in $D(H)$ if for each $y$ in $H(x), x \in \operatorname{int} H^{-1}(N+y)$ for any neighborhood $N$ of zero in $Y$. A relation $H$ is open at $y$ in $R(H)$ if for each $x$ in $H^{-1}(y), y \in$ int $H(N+x)$ for any neighborhood $N$ of zero in $X$. Then clearly $H$ is open at $y$ if and only if $H^{-1}$ is LSC at $y$. These matters are described in detail in $[\mathbf{1}, \mathbf{2}, \mathbf{3}, \mathbf{7}, \mathbf{9}]$.

Now suppose that $S$ is a convex cone in $Y$. We say $f: X \rightarrow Y$ is $S$-convex exactly when the relation $H_{f}(x):=f(x)+S$ is convex. Then, allowing $f(x)$ to be plus infinity, we have the domain of $f, \operatorname{dom}(f):=D\left(H_{f}\right)$. We recall that $S$ is generating if $S-S=Y$ and normal if there is a neighborhood base at 0 consisting of sets $V$ such that $(S-V) \cap(V-S)=V$. When $S$ is normal, $H_{f}$ is LSC at $x$ if and only if $f$ is continuous at $x$ [2]. If $S \subset X$ and $K \subset Y$ are convex cones, then we say $f$ is isotone with respect to $S$ and $K$ if $f\left(x_{1}\right)-f\left(x_{2}\right) \in K$ whenever $x_{1}-x_{2} \in S$ and $x_{1}, x_{2}$ are in $\operatorname{dom}(f)$. We recall that the core of a set $A, \operatorname{core}(A)$, is the set of points $\bar{a}$ in $A$ such that for all $x$ in $X, x \in \lambda(A-\bar{a})$ for $\lambda$ sufficiently large (depending on $x$ ). If $0 \in \operatorname{core}(A)$ we say $A$ is absorbing.

We denote the continuous dual cone of $S$ in $X$ by $S^{+}:=\left\{x^{\prime} \in X^{\prime} \mid x^{\prime}(x) \geq\right.$ 0 for $x$ in $S$ \}. Here $X^{\prime}$ is the continuous dual of $X$.

\section{The main result.}

DEFINITION 2.1. Let $X$ and $Y$ be linear spaces.

(a) Let $S \subset X$ be a convex cone. We say $H: X \rightarrow Y$ is an $S$-isotone relation if for $x$ in $X$

$$
H(x+S) \subset H(x),
$$

Received by the editors November 8, 1985.

1980 Mathematics Subject Classification (1985 Revision). Primary 46A30; Secondary 52A07, $49 \mathrm{D} 45$.

Research completed while the author was visiting U.E.R. des Sciences de Limoges. 
or equivalently, if $H\left(x_{1}\right) \subset H\left(x_{2}\right)$ whenever $x_{1} \geq_{S} x_{2}\left(x_{1}-x_{2} \in S\right)$.

(b) Let $S \subset Y$ be a convex cone. We say that $H: X \rightarrow Y$ is an $S$-invariant relation if for $x$ in $X$

$$
H(x)-S \subset H(x)
$$

Then $H$ is $S$-isotone if and only if $H^{-1}$ is $S$-invariant (reversing the roles of $X$ and $Y$ ).

THEOREM 2.2. Let $X$ and $Y$ be topological vector spaces. Let $H: X \rightarrow Y$ be a convex relation.

(a) Let $Y$ be complete and metrizable. Suppose that $H$ is $S$-invariant for a closed generating cone $S$ in $Y$. Then $H$ is open throughout the core of $R(H)$.

(b) Let $X$ be complete and metrizable. Suppose that $H$ is $S$-isotone for a closed generating cone $S$ in $X$. Then $H$ is LSC throughout the core of $D(H)$.

PROOF. From the previous discussion, we see that (a) and (b) are entirely dual (on replacing $H$ by $H^{-1}$ ). Thus we need prove only (a). Let $y_{0}$ lie in core $(R(H)$ ). Let $x_{0} \in H^{-1}\left(y_{0}\right)$. On replacing $H$ by $H\left(\cdot+x_{0}\right)-y_{0}$ [which leaves hypotheses and conclusions unchanged] we may suppose $x_{0}=0$ and $y_{0}=0$. Let $U$ be any absorbing subset of $X$. Then $H(U)$ is "almost absorbing" in $Y$. Indeed, since $H$ is convex and $0 \in H(0)$, for $u$ in $X$ we have

$$
2^{-n} H\left(2^{n} u\right) \subset 2^{-n} H\left(2^{n} u\right)+\left(1-2^{-n}\right) H(0) \subset H(u), \quad n \in \mathbf{N} .
$$

Thus

$$
R(H)=\bigcup_{\mathbf{N}} H\left(2^{n} U\right) \subset \bigcup_{\mathbf{N}} 2^{n} H(U)
$$

since $U$ is absorbing. As $R(H)$ is absorbing,

$$
Y=\bigcup_{n=1}^{\infty} 2^{n} H(U) \text {. }
$$

Let $\left(W_{n}\right)_{\mathbf{N}}$ be a neighborhood base at zero in $Y$ such that $W_{n+1}+W_{n+1} \subset W_{n}$ for $n$ in $\mathbf{N}$. Let

$$
V_{n}:=2^{-n}\left[W_{n} \cap S-S\right] .
$$

Then $V_{n}$ is a zero neighborhood. Indeed, the convex relation $Q: Y \rightarrow Y$ defined by $Q(y):=y-S$ if $y \in S$, is closed with $R(Q)=Y$. Since $Y$ is complete metrizable, the Fréchet open mapping theorem $[\mathbf{1}, \mathbf{4}]$ shows that $0 \in \operatorname{int} Q\left(W_{n}\right)$ for each $n$. This also follows from Klee's result [5, p. 194]. If some $V_{n}$ lies in $H(U)$ we are done.

If not, select $v_{n}$ in $V_{n}$ with $v_{n} \notin H(U)$ for each $n$. Then (2.4) shows that $v_{n} \leq_{S} 2^{-n} w_{n}$ for some $w_{n}$ in $W_{n} \cap S$. Let $z_{m}:=\sum_{n=0}^{m} w_{n}$.

Then inductively, $z_{m+p}-z_{m} \in W_{m} \cap S$ for $m$ and $p$ in N. Hence $\left(z_{n}\right)_{\mathbf{N}}$ is Cauchy and converges to some $z$ in $S$, as $S$ is closed. Now $2^{-n} z \geq_{S} 2^{-n} w_{n} \geq_{S} v_{n}$ and so $v_{n} \in 2^{-n} z-S$. Then (2.2) shows that $2^{-n} z \notin H(U)$. This contradicts (2.3). Thus $H$ is open at $y_{0}$.

REMARK 2.3. We have in fact established slightly more in (a): if $y_{0} \in$ core $(R(H))$, then

$$
y_{0} \in \operatorname{int} H\left(x_{0}+U\right)
$$

for any $x_{0}$ in $H^{-1}\left(y_{0}\right)$ and any absorbing set $U$ in $X$ (and similarly in (b)). 
Corollapy 2.4. Let $X$ and $Y$ be topological vector spaces. Suppose that $X$ is complete metrizable and $S \subset X$ is a closed generating convex cone. Suppose that $f: X \rightarrow Y \cup\{\infty\}$ is $K$-convex for some normal convex cone $K$ in $Y$. Suppose also that $f$ is isotone with respect to $S$ and $K$. Then $f$ is continuous throughout core $(\operatorname{dom}(f))$.

Proof. $H_{f}(x):=f(x)+K$ is convex and $S$-isotone. Thus Theorem 2.2(b) shows that $H_{f}$ is LSC on core $(D(H))$. Since $K$ is normal, $f$ is actually continuous on $\operatorname{core}(\operatorname{dom}(f))[\mathbf{2}$, p. 191].

We futher specialize to recover the Nachbin-Namioka result given by Peressini $[5$, p. 86].

Corollary 2.5. With $X, Y, S$, and $K$ as above, suppose that $P: X \rightarrow Y$ is linear and suppose that $P$ is positive: $P(S) \subset K$. Then $P$ is continuous.

Proof. $P$ is $K$-convex, isotone with respect to $S$ and $K$, and $\operatorname{dom}(P)=X$.

A similar result to Theorem 2.2 is easily obtained when int $S$ is nonempty.

Proposition 2.6. Suppose in Theorem 2.2 that int $S$ is nonempty, but not necessarily closed, while $X$ and $Y$ are arbitrary topological vector spaces. Then the conclusions of Theorem 2.2 hold.

ProOF. Again we show only (a). But now if $y_{0} \in H\left(x_{0}\right)$,

$$
y_{0}-\operatorname{int} S \subset H\left(x_{0}\right)-S \subset H\left(x_{0}\right) .
$$

It follows that int $H\left(x_{0}\right)$ is nonempty and that $H$ is (strongly) open at some point. Then $H$ is open throughout int $R(H)[2$, p. 191].

Similarly, all the corollaries still obtain.

COROLlaRY 2.7. Let $X$ and $Y$ be topological vector spaces with $Y$ complete metrizable. Let $C \subset X$ be a convex set. Let $f: X \rightarrow Y \cup\{\infty\}$ be $S$-convex with respect to a closed generating cone $S$.

(a) Suppose that

$$
0 \in \operatorname{core}[f(C)+S] \text {. }
$$

Then the convex relation $H$ defined by

$$
H(x):=\left\{\begin{array}{l}
f(x)+S, \quad x \in C, \\
\varnothing, \quad \text { else }
\end{array}\right.
$$

is open at zero.

(b) Let $p: X \rightarrow \mathbf{R} \cup\{\infty\}$ be convex with $H^{-1}(0) \cap$ core $(\operatorname{dom}(p)) \neq \varnothing$. Consider the convex program

$$
\text { (CP) }-\infty<\mu:=\inf \left\{p(x) \mid f(x) \leq_{S} 0, x \in C\right\} .
$$

Then Lagrange multipliers exist for $(\mathrm{CP})$ : there is a continuous linear functional $\lambda$ on $Y$ such that

$$
\mu=\inf \{p(x)+\lambda f(x) \mid x \in C\}
$$

and $\lambda(S) \geq 0$.

Proof. $H$ is convex and $(-S)$-invariant. This establishes (a) as a consequence of Theorem 2.2(a). 
(b) Let $0 \in H\left(x_{0}\right)$ with $x_{0}$ in core $(\operatorname{dom}(p))$. Then $U:=\left\{x \mid p\left(x+x_{0}\right) \leq p\left(x_{0}\right)+1\right\}$ is absorbing in $X$. Then (2.5) shows that $0 \in \operatorname{int} H\left(x_{0}+U\right)=: W$.

Let $h(w):=\inf \{p(x) \mid w \in H(x)\}$. Then, as always, $h$ is convex and also $h(w) \leq$ $p\left(x_{0}\right)+1$ for $w$ in $W$. Since $h(0)$ is finite, $h$ is continuous on $U$ and as in $[\mathbf{1}, \mathbf{8}]$ this suffices to establish (2.9).

Note that there are no continuity or closure hypotheses on $p, f$, or $C$, other than those implicit in the closedness of $S$.

COROLlaRY 2.8. Let $X$ and $Y$ be topological vector spaces with $Y$ complete metrizable. Let $K \subset X$ and $S \subset Y$ be convex cones with $S$ closed and generating. Let $A: X \rightarrow Y$ be linear (possibly not even densely defined).

(a) The following are equivalent:

$$
\begin{aligned}
& \text { (1) } A(K)-S=Y, \\
& \text { (2) } H_{A}(x):=\left\{\begin{array}{l}
A x-S, \quad x \in K, \\
\varnothing, \quad \text { else, }
\end{array}\right.
\end{aligned}
$$

is an open convex process.

(b) Both imply the Krein-Rutman identity [18]:

$$
\left[K \cap A^{-1}(S)\right]^{+}=K^{+}+S^{+} A .
$$

Proof. Clearly, $H_{A}$ is $S$-invariant and (a) follows.

(b) Suppose $\Phi \in\left[K \cap A^{-1}(S)\right]^{+}$. Then

$$
0=\inf \left\{\Phi(x) \mid-A x \leq_{S} 0, x \in K\right\} .
$$

The previous corollary applies and we can write

$$
0=\inf \{\Phi(x)-\lambda(A x) \mid x \in K\}
$$

for some $\lambda$ in $S^{+}$. But (2.13) shows $\Phi-\lambda A \in K^{+}$and so $\Phi \in K^{+}+S^{+} A$. The opposite inclusion always holds.

Note that we can phrase a similar result to (2.12) without assuming $\Phi$ is continuous.

Corollary 2.9. Let $X$ and $Y$ be as in Corollary 2.8. Let $H: X \rightarrow Y$ be $a$ convex process.

(a) Suppose that $H(0)$ contains a closed, generating subcone $S$. Then $H$ is open if and only if $R(H)=Y$.

(b) Suppose $H(0)$ is closed and $H(0)-H(0)=Y$. Then $H$ is open if and only if $R(H)=Y$.

ProOF. (a) We have

$$
H(x)+S \subset H(x)+H(0) \subset H(x)
$$

and $H$ is $(-S)$-invariant.

(b) In this case $H$ is $(-H(0))$-invariant.

Of course, Corollary 2.8(a) is a special case of Corollary 2.9(b). There is an obvious dual version.

EXAMPLE 2.10. (a) Let $X$ be the normed lattice, $\Phi$, consisting of all finite sequences in the supremum norm. Let $S:=K:=\left\{x \in X \mid x_{n} \geq 0, n \in \mathbf{N}\right\}$ be the 
lattice cone. Let $P: X \rightarrow X$ be defined by $(P x)_{n}:=n x_{n}$. Then $P$ is positive linear and discontinuous. All hypotheses of Corollary 2.4 hold other than completeness.

(b) Closed generating cones include dual cones of normal cones, closed cones with nonempty interior, and all closed lattice orderings. Thus our central hypothesis is generally satisfied.

COROLLARY 2.11. Let $Y$ be a complete metrizable topological vector space. Let $S \subset Y$ be a closed generating cone and let $V \subset Y$ be any absorbing set. Then the full hull of $V$,

$$
[V]_{S}:=(V-S) \cap(S-V)
$$

is a neighborhood of zero.

ProOF. $H(x):=x-S$ is convex and $S$-invariant on $Y$. Then Remark 2.3 shows that $0 \in \operatorname{int} H(V)$ and, by symmetry, $0 \in \operatorname{int}[H(V) \cap-H(V)]$.

With $\Phi, S$ as in Example 2.10(a) we let $V:=\left\{x \in \Phi|| x_{n} \mid \leq 2^{-n}, n \in \mathbf{N}\right\}$. Then $V$ is absorbing and $[V]_{S}=V$ has no interior.

There is also a local version of Theorem 2.2, which may be useful in applications. We say that $H$ is locally $S$-isotone around $x_{0}$ in $D(H)$ if there is a neighborhood $N$ of $x_{0}$ in $X$ such that $H\left(x_{1}\right) \subset H\left(x_{2}\right)$ when $x_{2} \leq_{S} x_{1}$ and $x_{1}, x_{2} \in \mathbf{N}$. Similarly, $H$ is locally $S$-invariant around $y_{0}$ in $R(H)$ if there is a neighborhood $N$ of $y_{0}$ in $Y$ such that if $y_{1} \in H(x)$ and $y_{2} \leq_{S} y_{1}$ with $y_{1}, y_{2}$ in $N$, then $y_{2} \in H(x)$. An analysis of the proof of Theorem 2.2 shows that (a) will prove that if $H$ is locally $S$-invariant around $y_{0}$ in core $R(H)$, then $H$ is open around $y_{0}$ (and similarly in (b)). It follows as in $[\mathbf{1}]$ that $H$ is actually open throughout core $(R(H))$.

In a lattice setting it is natural to consider the following variant of isotonicity. We say $H$ is $S$-absolutely isotone if

$$
H\left(x_{1}\right) \subset H\left(x_{2}\right) \text { whenever }\left|x_{2}\right| \leq_{S}\left|x_{1}\right| .
$$

This is equivalent to demanding that $H^{-1}(y)$ is solid for each $y$ : if $x_{1} \in H^{-1}(y)$ and $\left|x_{2}\right| \leq_{S}\left|x_{1}\right|$, then $x_{2} \in H^{-1}(y)$.

It is also easy to verify that $H$ is absolutely isotone exactly when

$$
H(x)=H(|x|) \text {, and } H\left(x_{1}\right) \subset H\left(x_{2}\right) \text { if } 0 \leq_{S} x_{2} \leq_{S} x_{1} .
$$

Moreover, if $H$ is $S$-isotone and convex, then $H(|\cdot|)$ is $S$-absolutely isotone and convex.

Theorem 2.2 has an obvious analogue in this context, in which we assume that $|\cdot|$ is continuous.

3. A bornological variant. Recall that a locally convex space is bornological if every symmetric convex subset which absorbs bounded sets is a zero neighborhood. Every metrizable space is bornological, as are inductive limits and quotients of bornological spaces [6]. A convex cone $S$ in $X$ is a strict b-cone if each bounded set $B$ in $X$ lies inside a set of the form $S \cap B^{\prime}-S \cap B^{\prime}$ for some other bounded set $B^{\prime}$. Normal and strict $b$-cones are effectively dual to each other [5]. We will say that $S$ is increasingly sequentially complete if every $S$-isotone Cauchy sequence converges. This holds in any complete metrizable space, or in any semireflexive space.

THEOREM 3.1. Let $X$ and $Y$ be locally convex spaces. Let $H: X \rightarrow Y$ be a convex relation. 
(a) Let $Y$ be bornological. Suppose that $H$ is $S$-invariant with respect to an increasingly sequentially complete strict b-cone $S$ in $Y$. Then $H$ is open throughout $\operatorname{core}(R(H))$.

(b) Let $X$ be bornological. Suppose that $H$ is $S$-isotone with respect to an increasingly sequentially complete strict b-cone $S$ in $X$. Then $H$ is continuous throughout core $(D(H))$.

Proof. Again (a) and (b) are equivalent and we will establish (a). As before we may assume that $y_{0}=0, x_{0}=0$, and $0 \in \operatorname{core}(R(H))$. Then let $U$ be any convex absorbing subset of $X$. Again, it suffices to show that $0 \in \operatorname{int} H(U)$. Now $H(U)$ is convex because $H$ and $U$ are. As $Y$ is real and bornological, it suffices to show that $H(U)$ absorbs bounded sets. Suppose not. Then we can find $B$ bounded and $\left(b_{n}\right)_{\mathbf{N}} \subset B$ such that

$$
4^{-n} b_{n} \notin H(U) \text { for } n \in \mathbf{N} \text {. }
$$

Since $S$ is a strict $b$-cone, $B \subset S \cap B_{0}-S$ for some bounded convex symmetric $B_{0}$. Let $\left(d_{n}\right)_{\mathrm{N}}$ lie in $S \cap B_{0}$ with $b_{n} \leq_{S} d_{n}$ for each $n$.

Let $z_{n}:=\sum_{m=0}^{n} 2^{-m} d_{m}$. Then

$$
z_{n+p}-z_{n} \in\left(2^{-n} B_{0}\right) \cap S
$$

for $n, p$ in $\mathbf{N}$. Thus $\left(z_{n}\right)_{\mathbf{N}}$ is Cauchy because $B_{0}$ is bounded, and increases. By assumption, $\left(z_{n}\right)$ converges to $z$ in $S$. Then

$$
2^{-n} z \geq_{S} 4^{-n} d_{n} \geq_{S} 4^{-n} b_{n} \quad \text { for } n \in \mathrm{N} .
$$

As $H$ is $S$-invariant, $2^{-n} z \notin H(U)$ for each $n$. This contradicts (2.3). Thus $H(U)$ does absorb bounded sets. then

REMARK. (a) As before we have established more in (a): If $y_{0} \in \operatorname{core}(R(H))$,

$$
y_{0} \in \operatorname{int} H\left(x_{0}+U\right)
$$

for any $x_{0}$ in $H^{-1}\left(y_{0}\right)$ and any convex, absorbing subset $U$.

(b) Thus all the corollaries of $\S 2$ remain valid under the appropriate hypotheses of Theorem 3.1. In particular Corollary 2.5, so adjusted, recaptures Schaefer's result for linear operators [5, p. 86]. In Corollary 2.11, $V$ must be supposed convex.

(c) As we have illustrated these open mapping results are applicable in a variety of situations. Thus, they should be a useful addition to the more standard closed graph type of results due to Robinson [7], Ursescu [9], Jameson [4], the author [1, $\mathbf{2}, \mathbf{3}]$, and elsewhere.

This is particularly so in the context of Corollaries $2.7,2.8$, and 2.9 because we make no hypotheses on the domain, only on the perturbation space. Moreover, the range space conditions are very often met.

\section{REFERENCES}

1. J. M. Borwein, Convex relations in optimization and analysis, Generalized Concavity in Optimization and Economics, Academic Press, New York 1981, pp. 335-377.

2. __ A Lagrange multiplier and a sandwich theorem for convex relations, Math. Scand. 48 (1981), 189-204.

3. _ Adjoint process duality, Math. Oper. Res. 8 (1983), 403-434.

4. G. J. Jameson, Convex series, Proc. Cambrige Philos. Soc. 72 (1972), 37-47. 
5. A. L. Peressini, Ordered topological vector spaces, Harper \& Row, New York, 1967.

6. A. P. Robertson and W. J. Robertson, Topological vector spaces, Cambridge Univ. Press, 1964.

7. S. Robinson, Regularity and stability for convex multivalued functions, Math. Oper. Res. 1 (1976), 130-143.

8. R. T. Rockafellar, Conjugate duality and optimization, SIAM, Philadelphia, Pa., 1974.

9. C. Ursescu, Multifunctions with convex closed graph, Czechoslovak Math. J. 7 (1975), 438-441.

Department of Mathematics, Dalhousie University, Halifax, Nova Scotia, CANADA B3H 3J5 